\title{
Miniaturkalorimeter mit integriertem Sensorarray
}

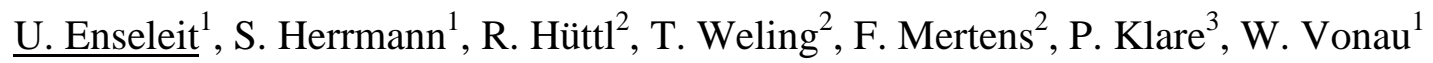 \\ ${ }^{1}$ Kurt-Schwabe-Institut für Mess- und Sensortechnik e.V. Meinsberg \\ Kurt-Schwabe-Straße 4, D-04720 Ziegra-Knobelsdorf, www.ksi-meinsberg.de \\ ${ }^{2}$ TU Bergakademie Freiberg, Institut für Physikalische Chemie, D-09599 Freiberg \\ ${ }^{3}$ IMM Ingenieurbüro GmbH, Leipziger Straße 32, D-09648 Mittweida
}

\section{Zusammenfassung}

Vorgestellt wird ein miniaturisiertes Reaktorsystem, welches durch eine kalorimetrische Reaktorzelle mit einem integrierten chemischen Sensorarray für Messungen in der Flüssigkeitsphase gekennzeichnet ist. Es gestattet die Durchführung chemischer und biotechnologischer Synthesen im Volumenmaßstab weniger Milliliter. Damit dient das System der Optimierung von Prozessabläufen, sowohl hinsichtlich der Auffindung optimaler physikalischer und chemischer als auch biotechnologischer Syntheseparameter.

\section{$1 \quad$ Einleitung}

Kalorimetrische Methoden werden für die Entwicklung, Optimierung und Kontrolle von chemischen und biologischen Prozessabläufen in jüngster Zeit verstärkt eingesetzt. Für klassische Anwendungen in der Sicherheitstechnik [1] beispielweise zur Risikoabschätzung des vorhandenen Energiepotenzials eines Reaktionsgemisches unter bestimmter Prozessführung oder zur Auslegung von Kühlkreisläufen in der Prozesstechnik werden zuverlässige Daten durch reaktionskalorimetrische Untersuchungen notwendig. Somit kann eine optimale Regelung eines Produktionsprozesses gewährleistet werden, ohne dass Gefahren für die Umwelt, in Form von Havarien durch Produktionsausfälle entstehen [2]. Durch eine optimale Prozessführung besteht ebenfalls die Möglichkeit, energieeffizient zu arbeiten und damit Kosten zu sparen.

\section{Elektrochemisches Sensorarray}

Schwerpunkt stellte die Entwicklung eines elektrochemischen Sensorarrays dar. Das angestrebte kleine Reaktorvolumen $(<25 \mathrm{~mL})$ im Miniaturkalorimeter erforderte die Realisierung der chemischen Sensorik in Form von planaren Sensoren, wozu die Dickschichttechnologie zum Einsatz kam. Besonders wichtig ist es gleichfalls, ein miniaturisiertes und dennoch genügend langzeitstabiles Referenzsystem für die potentiometrische Sensorik einzusetzen, welches nicht von der Zusammensetzung der zu untersuchenden Messlösungen

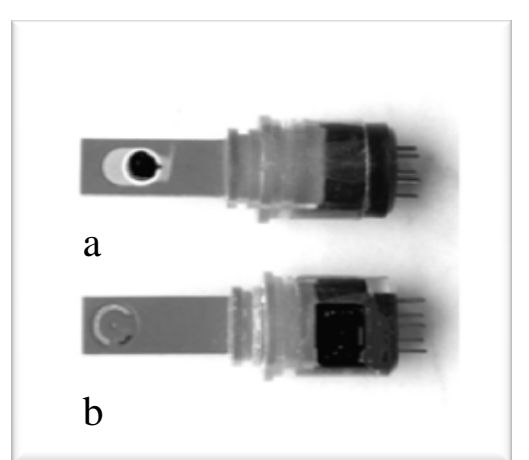
beeinflusst wird.

Das Sensorarray besteht im Einzelnen aus planaren Sensorstrukturen zur potentiometrischen $\mathrm{pH}$ - und Redoxpotenzialmessung auf der einen Seite des Sensors und aus einem membranbedeckten 3-Elektrodensystem zur amperometrischen Sauerstoffmessung auf der gegenüberliegenden Seite. Das Sensorsubstrat basiert auf einer $\mathrm{Al}_{2} \mathrm{O}_{3}$-Keramik (Bild 1).

Bild 1 Sensorarray des Miniaturkalorimeters mit Einbaustutzen in den Deckel des Reaktionsgefäßes und elektrischen Kontakten zum Elektronikmodul a) pH- und Redoxelektrode, b) Sauerstoffelektrode 


\section{$2.1 \quad$ pH- und Redoxsensor}

Die planare pH-Elektrode basiert auf einer pH-sensitiven Sb-Beschichtung von Gold- bzw. Graphitgrundstrukturen. Dabei wird aus einer antimonhaltigen Lösung elektrochemisch (kathodische Reduktion) eine dünne Antimonschicht, die an Luft oxidiert, abgeschieden. Zur Erprobung der Messfunktion der potentiometrischen pH-Sensoren wurden Messungen in Pufferlösungen ausgeführt. Die planare Redoxelektrode besteht aus Platin, das mit Hilfe einer kommerziell verfügbaren Pt-Paste auf das $\mathrm{Al}_{2} \mathrm{O}_{3}$-Keramik-Substrat aufgedruckt wurde.

Das Sensorarray wird vertikal von oben in das Messgut eintauchen, da die Durchmischung des Reaktorgutes über einen Magnetrührer am Gefäßboden erfolgt.

\subsection{Referenzsystem}

Die Funktionssicherheit aller potentiometrischen Sensoren wird entscheidend von der sicheren Funktion des Referenzelektrodensystems bestimmt.

Es ist Aufgabe des Referenzsystems, ein konstantes, von der Messlösung unabhängiges Potenzial vorzugeben. Gewöhnlich werden als Referenzsysteme Elektroden zweiter Art verwendet, die aus einem Metall bestehen, das mit einem seiner in Wasser schwerlöslichen Salze in Kontakt steht. Die Kombination aus beiden taucht in eine Lösung ein, die wiederum die Anionen des Salzes in bekannter Konzentration enthält. Die bekannteste und auch für die Anwendung geeignetste Elektrode ist die Silberchloridelektrode (Bild 2).

Neben der eigentlichen Referenzelektrode bestimmen der Referenzelektrolyt und das Diaphragma das Messverhalten des Referenzsystems. Als Referenzelektrolyt wird gesättigte KCl-Lösung verwendet.

Bild 2 Ag/AgCl, $\mathrm{KCl}_{\text {ges. }}$-Referenzsystem mit Einbaustutzen in den Deckel des Reaktorgefäßes und elektrischen Kontakten zum Elektronikmodul

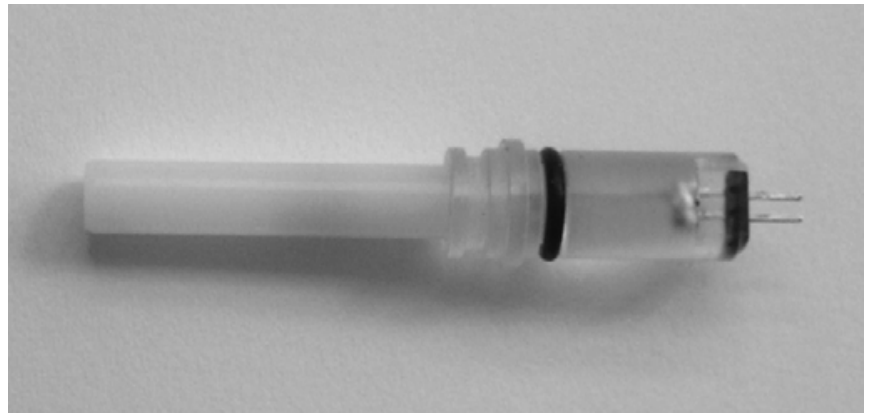

Eine leichte Bestückung bzw. Auswechslung der Referenzelektrode ist unkompliziert möglich. Sie taucht ebenfalls vertikal wie das Sensorarray in das Messgut im Reaktorgefäß ein. Nach ca. 100 ... 120 Tagen im Dauereinsatz müssten die Referenzelektroden durch Nachfüllen von gesättigter KCl-Lösung regeneriert werden. Es muss dafür Sorge getragen werden, dass sich das Diaphragma nicht durch Zellbewuchs zusetzt.

\subsection{Sauerstoffsensor}

Für die Sauerstoffsensoren wurde die Dreielektrodenanordnung mit Platin-Katode und Platin-Anode sowie Ag/AgCl-Referenzelektrode ausgewählt und als Sensorgrundstruktur eingesetzt. Die Elektrodenanordnung ist im Bild 3 gut erkennbar dargestellt.

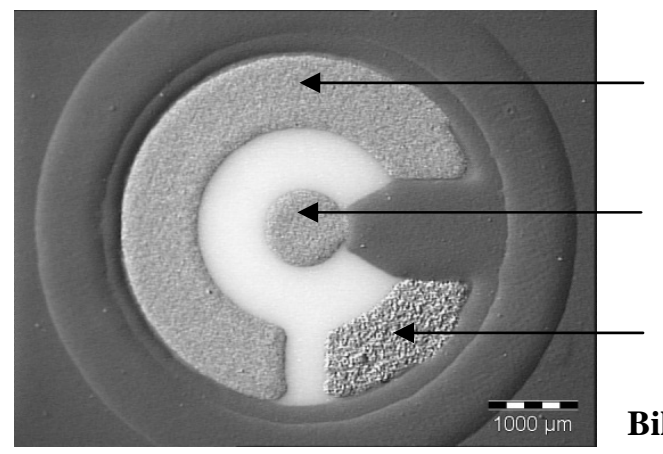

Gegenelektrode: Platin

Arbeitselektrode: Platin

Referenzelektrode: $\mathrm{Ag} / \mathrm{AgCl}$

Bild 3 Elektrodenanordnung des Sauerstoffsensors, Dreielektrodensystem 


\section{$3 \quad$ Miniaturkalorimeter}

Die Integration der miniaturisierten chemischen Sensorik erfordert einen nicht nur auf die Kulturbedingungen, sondern auch auf die Sensorik abgestimmten Aufbau der Reaktionszelle als zentralen Bestandteil des Miniaturkalorimeters. Das Reaktorgefäß hat ein Volumen von $25 \mathrm{~mL}$.

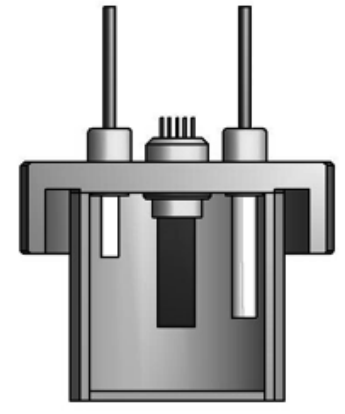

Bild 4 Computergrafik der Reaktorzelle mit integrierten Sensoren

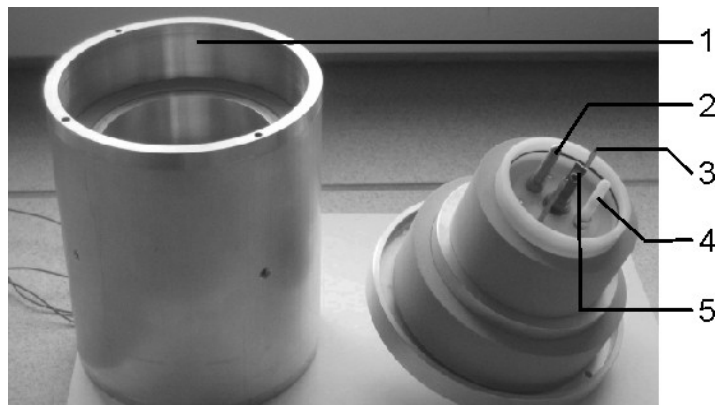

Bild 5 Reaktorgefäß; 1-Reaktor mit Isolation, 2-

Kalibrierheizer, 3- Kapillaren, 4- Referenzsystem, 5- Sensorar-

Bild 4 zeigt die Darstellung eines Musters der kalorimetrischen Reaktionszelle mit einem Außendurchmesser von $33 \mathrm{~mm}$ und einer Höhe von $43 \mathrm{~mm}$. In ihr sind die elektrochemischen Sensoren, der Rührer, die Edelstahlkapillaren zum Medientransport und die Heizung untergebracht. Bild 5 zeigt eine fotographische Aufnahme des Reaktorgefäßes mit Reaktordeckel.

Der Thermostat für das Miniaturkalorimeter (Bild 7) ist aufklappbar, um eine relativ einfache Montage des Systems im Betrieb zu gewährleisten. Seitlich befinden sich zwei Heizfolien. Um eine hohe Temperaturgenauigkeit zu erzielen, wurde die Regelung so konzipiert, dass jeweils zwei Peltierelemente und Heizer gegeneinander angebracht sind. Zur Vorthermostatisierung der Medien werden spiralförmig angeordnete Kapillarrohre in den Deckel eingebaut, die bis in die Reaktionskammer führen. In das Gesamtsystem wurden weiterhin eine Regel- und Steuereinheit, Heizer zur Leistungskompensation und elektrischen Kalibrierung sowie ein Magnetrührer integriert. Aus Bild 6 geht der modulare Gesamtaufbau hervor.

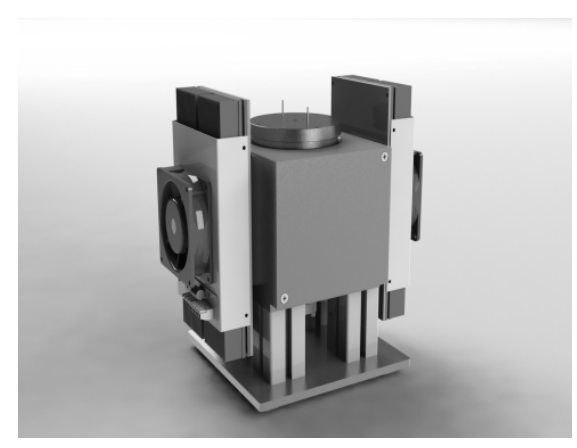

Bild 6 Computergrafik von Miniaturkalorimeter und Thermostat als Funktionseinheit

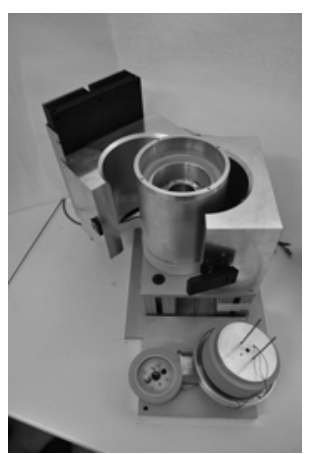

Bild 7 Miniaturkalorimeter mit integrierten Sensoren und Thermostat

\section{$4 \quad$ Testmessungen des Sensorarrays im Miniaturkalorimeter}

Die Testung des Sensorarrays, einschließlich der Referenzelektrode wurde in verschiedenen Bakterien- und Pilzkulturen, wie z.B. Pseudomonas putida oder Bäckerhefe (Saccharomyces cerevisiae) vorgenommen. Hefen zählen zu den bekanntesten Mikroorganismen in der Lebensmittelherstellung. Sie stellen eine wichtige Grundlage unter anderem im Brau- und Backgewerbe dar. Die gezielte Entwicklung von Hefen für die Lebensmittelindustrie ist deshalb von großer Bedeutung.

Bild 8 zeigt, wie die Sensoren auf Änderungen im Stoffwechsel ansprechen. Es wird der Zusammenhang zwischen pH-Wert, Sauerstoffgehalt und Redoxpotenzial nach Zuckerzugabe in eine Hefesuspension ersichtlich. 


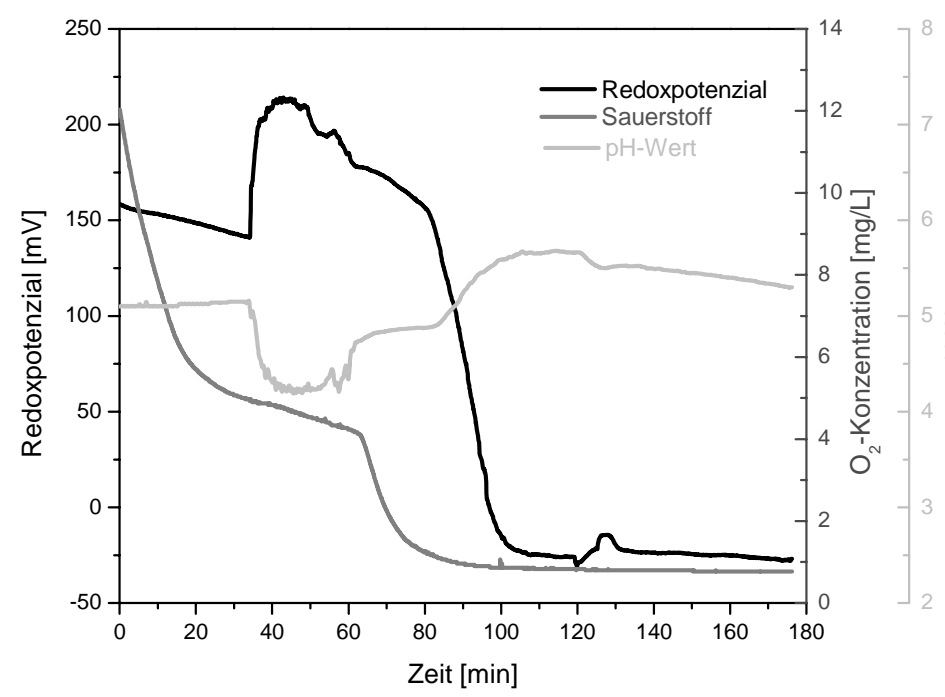

Bild 8 Verlauf von Redoxpotenzial, pH-Wert und Sauerstoffgehalt nach Zuckerzugabe (30 und 60 min) in einer Hefesuspension

Der Sauerstoffgehalt des Mediums nimmt deutlich ab, da der Zucker unter Sauerstoffverbrauch von den Hefezellen abgebaut wird. Das Redoxpotenzial und der $\mathrm{pH}$-Wert bleiben bis zur 1. Zuckerzugabe relativ konstant. Danach kann man eine Redoxpotenzialzunahme beobachten und anschließend einen starken Abfall. Der pH-Wert dagegen fällt durch die 1. Zuckerzugabe sprunghaft von ca. $\mathrm{pH}$ 5,75 auf pH 4,85 ab und ver ringerte sich jedoch nach der 2 . Zuckerzugabe kaum noch.

\section{$5 \quad$ Anwendungsmöglichkeiten}

Die Anwendungen des Miniaturkalorimeters liegen vor allem im Bereich der Biotechnologie und Pharmazie, speziell für den Einsatz im Labor, im Technikum oder in Pilotanlagen. Das Miniaturkalorimeter dient zur Kultivierung von Mikroorganismen oder zur Herstellung von Produkten im Miniaturmaßstab. Es zeichnet sich durch die Möglichkeit einer umfassenden Prozesskontrolle aus.

Die entwickelte miniaturisierte elektrochemische Sensorik, die in erster Linie auf das Miniaturkalorimeter und Anwendungen in der Biotechnologie zugeschnitten ist, lassen sich darüber hinaus auch für zahlreiche andere Applikationen, bei denen Online-Bestimmungen von Analytkonzentrationen in kleinsten Volumina eine Rolle spielen, nutzen.

\section{Danksagung}

Das Vorhaben wurde gefördert aus Mitteln des Europäischen Fonds für regionale Entwicklung (EFRE) und mit Mitteln des Freistaates Sachsen (SAB) im Rahmen des Förderprogrammes „Innovative technologieorientierte Verbundprojekte auf dem Gebiet der Zukunftstechnologien im Freistaat Sachsen“ unter dem Förderkennzeichen 12600/2078.

\section{$7 \quad$ Literatur}

[1] Westerterp, K. R.; Molga, E. J.: Safety and runaway prevention in batch and semibatch reactors - a review: Chemical Engineering Research and Design, 84(A7) (2006) 543-552

[2] Marison, I. W; Liu, J.-S.; Ampuero,S.; von Stockar, U.; Schenker, B.: Biological reaction calorimetry: Development of high sensitivity bio-calorimeters. Thermochimica Acta, 309 (1998) 157-173 